\title{
Modulation of expression of the nuclear receptor NROB2 (small heterodimer partner I) and its impact on proliferation of renal carcinoma cells
}

\author{
Katharina Prestin ${ }^{1, *}$ \\ Maria Olbert ${ }^{2, *}$ \\ Janine Hussner' \\ Tamara L Isenegger' \\ Daniel G Gliesche' \\ Kerstin Böttcher ${ }^{2}$ \\ Uwe Zimmermann ${ }^{3}$ \\ Henriette E Meyer zu \\ Schwabedissen' \\ 'Department of Pharmaceutical \\ Sciences, Biopharmacy, University \\ of Basel, Basel, Switzerland; \\ ${ }^{2}$ Center of Drug Absorption and \\ Transport, Institute of Pharmacology, \\ ${ }^{3}$ Department of Urology, \\ University Medicine Greifswald, \\ Greifswald, Germany \\ *These authors contributed equally to \\ this work
}

This article was published in the following Dove Press journal:

OncoTargets and Therapy

8 August 2016

Number of times this article has been viewed
Abstract: Mammalian nuclear receptors (NRs) are transcription factors regulating the expression of target genes that play an important role in drug metabolism, transport, and cellular signaling pathways. The orphan and structurally unique receptor small heterodimer partner 1 (syn NR0B2) is not only known for its modulation of drug response, but has also been reported to be involved in hepatocellular carcinogenesis. Indeed, previous studies show that NR0B2 is downregulated in human hepatocellular carcinoma, suggesting that NR0B2 acts as a tumor suppressor via inhibition of cellular growth and activation of apoptosis in this tumor entity. The aim of our study was to elucidate whether NR0B2 may also play a role in other tumor entities. Comparing NR0B2 expression in renal cell carcinoma and adjacent nonmalignant transformed tissue revealed significant downregulation in vivo. Additionally, the impact of heterologous expression of NR0B2 on cell cycle progression and proliferation in cells of renal origin was characterized. Monitoring fluorescence intensity of resazurin turnover in RCC-EW cells revealed no significant differences in metabolic activity in the presence of NR0B2. However, there was a significant decrease of cellular proliferation in cells overexpressing this NR, and NR0B2 was more efficient than currently used antiproliferative agents. Furthermore, flow cytometry analysis showed that heterologous overexpression of NR0B2 significantly reduced the amount of cells passing the $\mathrm{G} 1$ phase, while on the other hand, more cells in $\mathrm{S} / \mathrm{G} 2$ phase were detected. Taken together, our data suggest that downregulation of NR0B2 may also play a role in renal cell carcinoma development and progression.

Keywords: small heterodimer partner, SHP1, NR0B2, nuclear receptor, kidney cancer, proliferation

\section{Introduction}

In the past decade, the mammalian nuclear receptor (NR) superfamily of transcription factors has been the focus of numerous studies analyzing their role in physiology and pathophysiology, revealing their key function in fundamental cellular processes, including metabolism and proliferation. ${ }^{1,2}$ One of the outstanding characteristics of NRs is the capacity to regulate the expression of a variety of genes, thereby coordinating transcription and function of gene networks. In detail, members of this protein family display three characteristic domains, namely the activation function, a highly conserved zinc-finger region for DNA binding, and a large hydrophobic domain mediating ligand binding and protein dimerization. ${ }^{3}$ Binding of specific activating ligands results in conformational changes controlling dimerization and affinity to specific DNA sequences in the regulatory regions of their targets. However, when talking about NRs, one has to consider an extraordinary member of this protein superfamily, which is the small
Correspondence: Henriette E Meyer zu Schwabedissen

Department of Pharmaceutical Sciences, Biopharmacy, University of Basel,

Klingelbergstrasse 50, CH-4056 Basel, Switzerland

Tel +4I 612671495

Fax +4| 6| 267 |498

Email h.meyerzuschwabedissen@unibas.ch 
heterodimer partner SHP1, also known as NR subfamily 0 group B member 2 (NR0B2). ${ }^{4}$

This particular NR is not only expressed in various human organs with outstanding importance in metabolic homeostasis, such as the liver, pancreas, and kidneys, but is also assumed to be a major regulator of several metabolic pathways, including glucose, bile acid, cholesterol, and fatty acid homeostasis as reviewed by Kim et al. ${ }^{5}$

Importantly, the diversity of processes modulated by NR0B2 is the result of the unique functionality of this particular member of the superfamily. In detail, NR0B2 is an atypical NR, as it lacks the conserved zinc-finger DNA-binding domain. ${ }^{4}$ Therefore, NR0B2 is not capable of binding directly to response elements to regulate its target genes. Instead, NR0B2 interacts with various members of the superfamily of NRs, which in turn contain a DNA-binding domain and thereby exhibit transcriptional regulation. ${ }^{4}$ In this way, NR0B2 is assumed to function as a transcriptional corepressor, inhibiting the activity of its binding partners by coactivator competition, recruitment of corepressors, or direct interaction with their DNA-binding domain. ${ }^{6}$

More precisely, compared to NR-binding proteins harboring LXXLL-motifs in their amino acid sequence, the NR0B2 contains two functional LXXLL-related motifs located in helix 1 of its N-terminal ligand-binding domain and in helix 5 of its carboxy-terminus, which target the activation function (AF-2) of other NRs, such as peroxisome proliferator-activated receptor gamma (NR1C3), aryl hydrocarbon receptor, constitutive androstane receptor (NR1I3), liver receptor homolog-1 (NR5A2), liver X receptor alpha (NR1H3), farnesoid X receptor (NR1H4), and pregnane $\mathrm{X}$ receptor (NR1I2). ${ }^{7-9}$ All these NRs are involved in transcriptional regulation of more or less well-defined gene networks, which explains the diversity of cellular functions modulated by NR0B2. ${ }^{7-9}$ Hence, NR0B2 not only influences physiological metabolic pathways, complex networks of drug metabolizing enzymes, and efflux transporters, but also carcinogenesis and tumor progression. . $^{3,10}$

Especially, NR0B2 is assumed to be involved in the regulation of cell cycle progression and apoptosis in liver. ${ }^{11,12}$ Indeed, Zhang et al reported that $\mathrm{NrOb2}^{-/-}$knockout mice lacking the expression of this NR spontaneously develop hepatocellular carcinoma (HCC) at the age of 12-15 months, which is accompanied by massive cellular proliferation and enhanced malignant transformation of hepatocytes. ${ }^{13}$ Moreover, a link between NR0B2 and tumor suppression was confirmed by findings in human tissue samples of HCC, showing not only significant downregulation of NR0B2 in hepatic tumor compared with nonmalignant transformed liver tissue, but also diminished expression of NR0B2 target gene sets involved in cell proliferation and metabolism. ${ }^{14-16}$

Even though there is rising evidence that NR0B2 functions as a tumor suppressor, little is known about the role of this NR in human tumors other than HCC. The aim of the present study was to determine whether NR0B2 downregulation can also be observed in other tumor entities of human organism.

\section{Materials and methods Human tissue samples}

Human renal tissue samples were obtained from patients undergoing surgery after diagnosis of renal cell carcinoma. The study was approved by the local ethics committee at the Medical Faculty of the University of Greifswald (III UV 12/03). Written informed patient consent was also obtained. After surgical resection, the malignant and nonmalignant transformed samples were cut into small pieces, snap frozen in liquid nitrogen, and stored at $-80^{\circ} \mathrm{C}$ until further use. For pulverization of the samples, $100 \mathrm{mg}$ of snap frozen tissues were grinded for 1 minute at $25 \mathrm{~Hz}$ in a mixer mill (MM400, Retsch, Haan, Germany) using a $5 \mathrm{~mL}$-grinding jar, which was cooled in liquid nitrogen before. A polymerase chain reaction (PCR)-ready first-strand cDNA panel containing normal and malignant samples of various tissues was commercially obtained from OriGene, Rockville, MD, USA (TissueScan $^{\mathrm{TM}}$ disease tissue quantitaPCR arrays CSRT101 and CSRT103/303/503, used for analysis in Figure 1).

\section{Cell lines}

For the present study, the renal carcinoma cell lines RCC-EW (KTCTL-2, CLS 300246, Heidelberg, Germany), Caki-1 (ATCC ${ }^{\circledR}$ HTB-46 $^{\mathrm{TM}}$ ), and Caki-2 (ATCC HTB-47) cells were used. The cell lines were kindly provided by Prof Nicole Endlich (Greifswald, Germany). Cell culture of RCC-EW cells was performed using Dulbecco's Modified Eagle's Medium supplemented with 10\% fetal calf serum (FCS) and 1\% GlutaMAX ${ }^{\mathrm{TM}}-1$ (each from LuBio Sciences, Lucerne, Switzerland) at $37^{\circ} \mathrm{C}$ with $5 \% \mathrm{CO}_{2}$ in a humidified atmosphere. Caki-1 and Caki- 2 cells were cultured under the same conditions in McCoy's 5A (modified) medium (LuBio Sciences) supplemented with $10 \%$ FCS.

\section{RNA extraction and cDNA synthesis}

Human adult tissue total mRNA extracted from liver and kidney was purchased from BioCat (Heidelberg, Germany). Extraction and DNase treatment of total RNA from frozen 
A

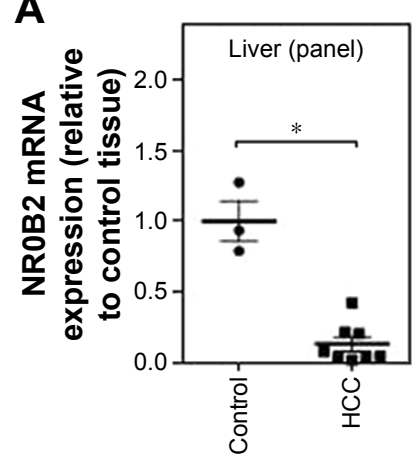

B

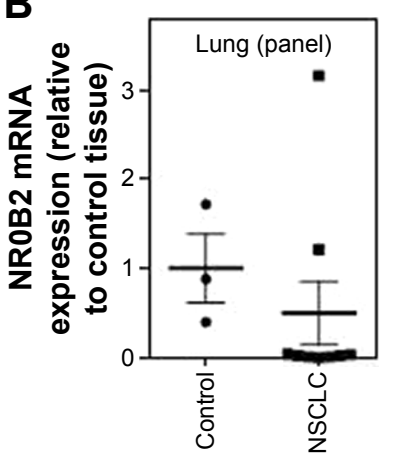

C

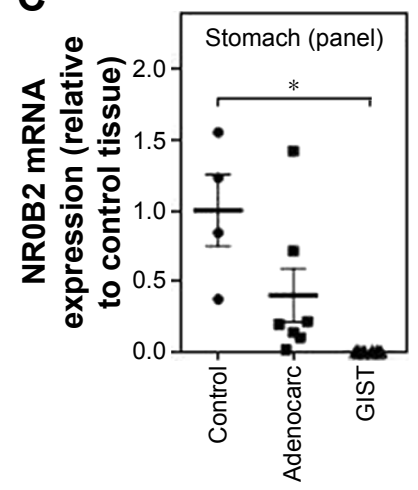

D

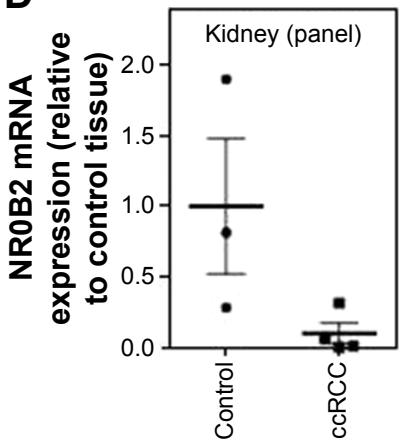

E

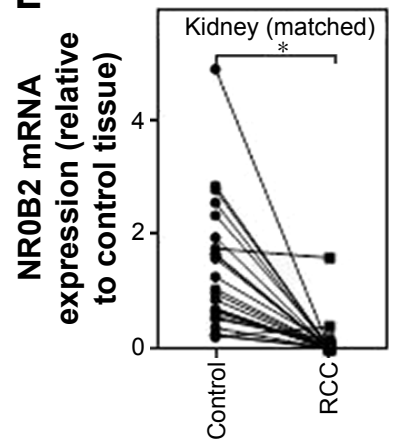

$\mathbf{F}$

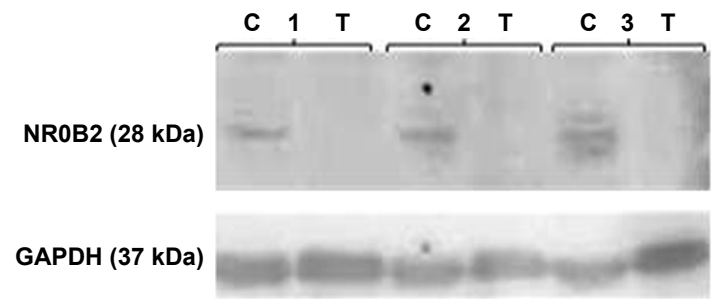

Figure I NROB2 expression in tumor tissue.

Notes: The mRNA expression of NROB2 was assessed by TaqMan ${ }^{\circledR}$ quantitative RT-PCR comparing malignant and nonmalignant transformed tissue samples, (A-D) using the commercially available cDNA panel CSRTIO3 (includes stomach, adenocarcinoma of stomach, and GIST) and CSRTIOI (includes liver and HCC [stages I-IV], from lung and NSCLC [stages I-IV], kidney and ccRCC [stages I, III, and IV]) (Origene, Rockville, MD, USA), (E) or using samples of 24 individuals undergoing renal surgery after diagnosis of RCC. Data are presented as mean \pm SD and were obtained after performing the $2^{-\Delta \Delta C t}$ method $(* P<0.05$ by Mann-Whitney test). (F) Protein expression assessed by Western blot analysis comparing surgery samples of RCC tissue compared to adjacent healthy control tissue. GAPDH served as loading control.

Abbreviations: adenocarc, adenocarcinoma; c, control; ccRCC, clear cell renal cell carcinoma; GAPDH, glyceraldehyde 3-phosphate dehydrogenase; GIST, gastrointestinal stromal tumor; HCC, hepatocellular carcinoma; NROB2, nuclear receptor subfamily 0 group B member 2; NSCLC, non-small cell lung carcinoma; RT-PCR, real-time polymerase chain reaction; SD, standard deviation; T, tumor.

and pulverized renal human tissues and from cultured cells was conducted using the NucleoSpin ${ }^{\circledR}$ silica-membrane technology provided by Macherey-Nagel (Dueren, Germany) according to the manufacturer's instructions. After priming with random hexamer oligonucleotides, total RNA $(2 \mu \mathrm{g})$ was reverse transcribed using the high-capacity reverse transcription kit (LuBio Sciences).

\section{$\mathrm{TaqMan}^{\circledR}$ quantitative real-time PCR}

TaqMan quantitative real-time PCR (RT-PCR) was performed using cDNA (an equivalent of 10 ng RNA), TaqMan Gene Expression Master Mix, predeveloped TaqMan probes (Hs00222677_m1 for NR0B2, Hs00765553_m1 for cyclin D1/CCND1, and assay 4319413E for 18S ribosomal RNA), and the ViiA7TM RT-PCR system (each from LuBio Sciences). Expression was normalized to that of $18 \mathrm{~S}$ ribosomal RNA using the $\Delta \mathrm{Ct}$ method, where $\mathrm{Ct}$ is the threshold cycle. Relative expression was determined according to the $2^{-\Delta \Delta C t}$ method referring to the expression of the indicated control.

\section{Preparation of an NROB2-encoding adenovirus}

The coding sequence of NR0B2 (NM_021969.2) was amplified by PCR using the sense and antisense primer $5^{\prime}$-CTTG AGATGAGCACCAGCCAACCAGGGG- ${ }^{\prime}$ and $5^{\prime}$-CATG TCCCCAAAACAGGTCACCTGAGCAAAAGC-3', and subcloned into eukaryotic expression vector $\mathrm{pEF} 6 / \mathrm{V} 5$-His by TOPO ${ }^{\circledR}$ Cloning Reaction using the pEF6/V5-His TOPO TA expression kit (LuBio Sciences) according to the manufacturer's instructions.

The coding sequence of NR0B2 in pEF6/V5-HIS was then transferred into pENTR1A ${ }^{\circledR}$ (LuBio Sciences) using the FastDigest ${ }^{\mathrm{TM}}$ restriction enzymes KpnI and Not $\mathrm{I}$ (Thermo Scientific, Reinach, Switzerland). After ligation, amplification in Escherichia coli, and sequence control, the NR0B2 insert was transferred into $\mathrm{pAd} / \mathrm{CMV} / \mathrm{V} 5-\mathrm{DEST}^{\mathrm{TM}}$ (LuBio Sciences) by recombination using the Gateway ${ }^{\circledR}$ LR Clonase ${ }^{\mathrm{TM}} \mathrm{Kit}$ (LuBio Sciences). The adenoviral vector was digested with PacI and subsequently transfected 
into replication-competent HEK293A cells (Vira Power, LuBio Sciences, ATCC ${ }^{\circledR}$ Number CRL-1573). Amplification of the virus was determined by visualization of cell lysis by light microscopy. Afterward, the supernatant was used for further virus amplification in HEK293A cells. The amount of plaque forming units (pfu) was determined by 3-(4,5-dimethylthiazol-2-yl)-2,5-diphenyltetrazolium bromide plaque assay in HEK293A cells, as recommended by the manufacturer (LuBio Sciences). The adenoviral vector $\mathrm{pAd} / \mathrm{CMV} / \mathrm{V} 5-\mathrm{GW} / \mathrm{LacZ}$ served as a control in the experiments, as recommended by the manufacturer (LuBio Sciences).

\section{Protein preparation}

Preparation of tissue lysates was performed by resuspension of pulverized tissue or cultured cells in ice-cold radioimmunoprecipitation assay buffer (Sigma-Aldrich, Buchs, Switzerland) containing $50 \mathrm{mM}$ Tris- $\mathrm{HCl}$ of $\mathrm{pH} 8.0,150 \mathrm{mM}$ $\mathrm{NaCl}, 1 \%$ Igepal CA-630/NP-40, 0.5\% sodium deoxycholate, $0.1 \%$ sodium dodecyl sulfate, and 1:100 protease inhibitor cocktail (Sigma-Aldrich). After syringing the suspension with a 20-gauge needle and 15 minutes incubation on ice, cells were centrifuged 15 minutes at $13,000 \times g$ for removal of cell debris. The supernatant was stored at $-80^{\circ} \mathrm{C}$ until further use. The Pierce ${ }^{\circledR}$ BCA protein assay kit (Thermo Scientific) and an Infinite ${ }^{\circledR}$ M200 microplate reader (Tecan, Männedorf, Switzerland) were used for protein quantification.

\section{Immunodetection}

Tissue and cell lysates were used for immunodetection of NR0B2 by performing Western blot analysis. After sodium dodecyl sulfate polyacrylamide gel electrophoresis using $10 \%$ polyacrylamide gel, the proteins were electrotransferred to a Protran ${ }^{\circledR}$ nitrocellulose membrane (GE Healthcare, Glattbrugg, Switzerland) using the Mini-PROTEAN ${ }^{\circledR}$ Tetra Cell (Bio-Rad, Cressier, Switzerland). For blocking of unspecific binding, membranes were incubated with trisbuffered saline containing $0.04 \%$ Tween ${ }^{\circledR} 20$ containing 5\% albumin fraction V (Carl Roth, Arlesheim, Switzerland). NR0B2 protein was detected using a 1:1,000 dilution of rabbit polyclonal anti-NR0B2 antibody (ab96605) (Abcam, Cambridge, UK) and a horseradish peroxidase-labeled secondary goat anti-rabbit IgG antibody (Bio-Rad), each diluted in tris-buffered saline containing 0.04\% Tween 20 containing albumin fraction $\mathrm{V}$, Pierce ${ }^{\mathrm{TM}}$ ECL 2 Western blotting substrate (Thermo Scientific), and ChemiDoc ${ }^{\mathrm{TM}}$ XRS System (Bio-Rad). For immunofluorescence detection of NR0B2 in cell lines, a rabbit antihuman polyclonal antibody LS-B3560-50 (1:50 diluted in phosphate-buffered saline [PBS] containing 5\% FCS, LifeSpan BioSciences, Seattle, WA, USA) and Alexa Fluor ${ }^{\circledR}$ 488-labeled chicken anti-rabbit (1:300 diluted in PBS containing 5\% FCS, H+L A21200, Thermo Scientific) were used. Immunofluorescent staining of MKI67 was conducted using monoclonal rabbit anti-MKI67 antibody (1:25, DRM004, San Diego, CA, USA) and Alexa Fluor 488-coupled antibody (1:150). Cells were fixed with $4 \%$ paraformaldehyde and permeabilized with $0.2 \%$ Tween 20 for 10 minutes at room temperature. Samples were mounted using Roti ${ }^{\circledR}$-Mount FluorCare (Carl Roth) containing 4',6-diamidino-2-pheynylindole for nuclei staining. Staining was visualized with a Leica DMi8 microscope using a DFC365FX camera. MKI67 expression was quantified with the Leica Application Suite version 4.4 (Leica, Heerbrugg, Switzerland). Intensity values were determined by subtracting mean intensity value of regions that mark the background from the mean intensity values of regions with specific staining (using the channel detecting emission of Alexa Fluor 488 as gray scale). The resulting intensity values were normalized to the background-cleared average value of signal intensity of 4',6-diamidino-2-pheynylindole staining detected in the same marked regions. Five photographs per sample were analyzed. Staining was performed in three experiments.

\section{Hematoxylin and eosin staining}

Prior to staining of RCC-EW with hematoxylin and eosin (H\&E), $3.2 \times 10^{4}$ cells per well of a 12 -well plate were seeded on cover slips and treated for 96 hours with adenovirus. Afterward, the cells were washed with PBS, fixed with methanol:acetone $(1: 1)$ for 10 minutes at $-20^{\circ} \mathrm{C}$. For H\&E staining, the fixed cells were incubated with hematoxylin solution modified according to Gill II (Carl Roth) for 15 minutes, rinsed with tap water, incubated with eosin solution (Carl Roth, $75 \mathrm{mg}$ eosin in acidic 75\% ethanol) for 1 minute, and dehydrated in ascending ethanol concentrations (from $80 \%$ to $100 \%$ ). After mounting the cover slips on slides using Roti Histokit (Carl Roth), staining was visualized with a Leica DMi8 microscope using a MC170HD camera and the Leica Application Suite version 4.4.

\section{Determination of cell proliferation by cell counting}

Counting of RCC-EW cells was performed using the CASY ${ }^{\circledR}$ Model TT System (Roche Diagnostics, Indianapolis, IN, USA) based on the principle of electrical current exclusion. Briefly, $3.2 \times 10^{4}$ cells were seeded in a 12 -well plate and 
transduced with adenovirus (Ad)-NR0B2, or Ad-LacZ as control ( $50 \mathrm{pfu} / \mathrm{cell})$. Ninety-six hours after transfection, the cells were counted by aspirating $400 \mu \mathrm{L}$ of the cell suspension through a measuring capillary sized $150 \mu \mathrm{m}$ after trypsinization $(300 \mu \mathrm{L}$ trypsin-ethylenediaminetetraacetic acid 0.05\% per well, reaction stopped by $700 \mu \mathrm{L}$ Dulbecco's Modified Eagle's Medium per well) and diluted 1:200 in an isotonic liquid (CASYton, Roche Diagnostics). Caki-1 cells were defined as viable between sizes of 7.5-60.0 $\mu \mathrm{m}$ using the manufacturer's protocol "Setting Up An Animal Cell line". Sizes of viable Caki-2 and RCC-EW cells ranged between 7.75 and $40.0 \mu \mathrm{m}$.

\section{Determination of cell viability}

Cells were cultured in a 48 -well plate in $100 \mu \mathrm{L}$ of medium at $37^{\circ} \mathrm{C}$ for indicated periods of time. For background control, medium without cells was used. Then $10 \mu \mathrm{L}$ of resazurin solution (PromoKine, Heidelberg, Germany) were added to each well. After 3 hours, fluorescence intensity was monitored, measuring the excitation at $530 \mathrm{~nm}$ and emission at $590 \mathrm{~nm}$ using an Infinite M200 plate reader and i-control ${ }^{\mathrm{TM}}$ software (Tecan).

\section{Cell cycle analysis}

For cell cycle analysis, $1 \times 10^{5}$ cells/well were seeded in a sixwell plate and cultured for 24 hours. Afterward, the cells were incubated with medium containing Ad-NR0B2, or Ad-LacZ as control (50 pfu/cell) for 12 hours. At indicated time points, the cells were harvested by trypsinization and fixed for 24 hours in $70 \%$ ethanol at $4^{\circ} \mathrm{C}$. The cells were stained by 30 minutes incubation with PBS-containing propidium iodide $(50 \mu \mathrm{g} / \mathrm{mL})$, RNAse A ( $2 \mu \mathrm{g} / \mathrm{mL})$, and glucose $(15 \%$ $\mathrm{w} / \mathrm{v})$. Resuspended and propidium iodide-stained cells were analyzed using fluorescence-activated cell sorting system FACSCalibur $^{\mathrm{TM}}$ and the instrument software CellQuest ${ }^{\mathrm{TM}}$ (BD Biosciences, Heidelberg, Germany).

\section{Statistical analysis}

Statistical calculations were performed using GraphPad Prism 6 version 6.04 (GraphPad Software Inc., La Jolla, CA, USA).

\section{Results}

\section{Detection of NROB2 in malignant and nonmalignant transformed human tissues}

As it has previously been reported that NR0B2 expression is decreased in $\mathrm{HCC},{ }^{15,16}$ we investigated whether malignant transformation of other tissues is also associated with a regulation of NR0B2 mRNA levels. A first screening was performed assessing the amount of mRNA by TaqMan RT-PCR using a commercially available PCR-ready first-strand cDNA panel that contained samples of different tumors.

NR0B2 expression was significantly lower in hepatic tumor tissue compared to the nonmalignant transformed control tissue (relative expression $0.1 \pm 0.1$ vs $1.0 \pm 0.2$, MannWhitney test, $P<0.05$, Figure 1A). Furthermore, comparison of malignant and nonmalignant transformed tissue samples of lung (relative expression $0.5 \pm 1.1$ vs $1.0 \pm 0.7, P=0.14$, Figure 1B) showed a difference in NR0B2 expression, however, without statistical significance. There was also a trend for downregulation of NR0B2 in the tumor tissue of the stomach (relative expression in adenocarcinoma $0.4 \pm 0.5$ vs $1.0 \pm 0.5, P=0.07$, Figure $1 \mathrm{C}$ ), while NR0B2 expression was not detected in the gastrointestinal stromal tumor (GIST) of the stomach. Moreover, NR0B2 expression was reduced in renal cell carcinoma (Figure 1D). Despite the very small sample size in this first assessment of expression using the cDNA panel for screening, we verified our preliminary notion of downregulation of the NR by performing TaqMan quantitative RT-PCR using a collection of 24 samples of renal cell carcinoma obtained from different individuals and paired adjacent nonmalignant transformed renal tissue (relative expression $0.1 \pm 0.3$ vs $1.4 \pm 1.1$, Mann-Whitney test, $P<0.0001$, Figure 1E). In addition, performing Western blot analyses confirmed that NR0B2 expression in human specimen of renal cell carcinoma was significantly lower compared to nonmalignant transformed tissue, suggesting that the previously observed reduction of this NR may also play a role in this tumor entity (Figure 1F).

\section{Characterization of NROB2 expression and proliferation of cell lines isolated from renal carcinoma}

To identify a cellular model that could be used for a mechanistic study to evaluate the impact of NR0B2 on proliferation of renal carcinoma cells, we characterized the NR0B2 expression in a selection of immortalized renal carcinoma cell lines.

Performing quantitative RT-PCR showed that, compared to human kidney $(100.2 \% \pm 9.7 \%)$, NR0B2 mRNA expression was similar in human liver $(84.1 \% \pm 0.2 \%)$, whereas its expression was much lower in renal clear cell carcinoma cell lines Caki-1 and Caki-2 (relative expression referring to human kidney $0.1 \% \pm 0.0 \%$, and $8.0 \% \pm 3.5 \%$, respectively), and in the epithelial renal adenocarcinoma cell line RCC-EW $(30.3 \% \pm 7.0 \%)$ (Figure 2A). 
A

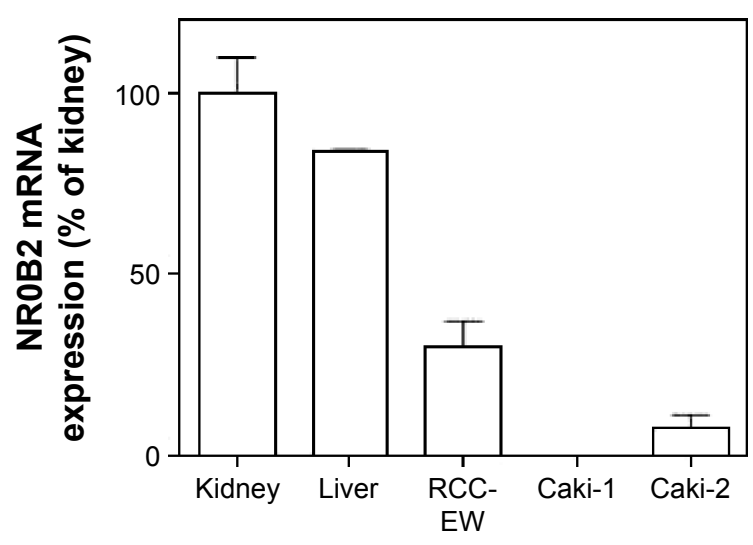

B

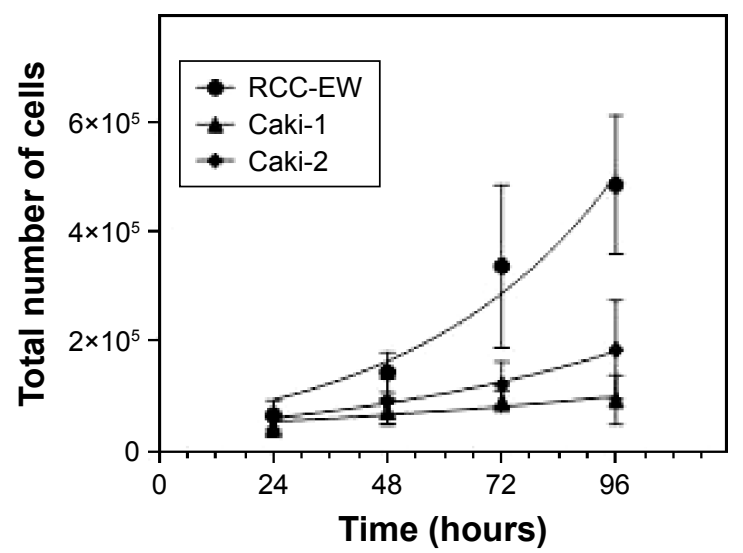

Figure 2 Characterization of NROB2 expression human renal carcinoma cell lines.

Notes: (A) NROB2 mRNA expression assessed by TaqMan ${ }^{\circledast}$ quantitative RT-PCR. Data are presented as mean \pm SD and were obtained after performing the $2^{-\Delta A C t}$ method referring the expression to that of human kidney (BioCat, Heidelberg, Germany). (B) For characterization of proliferation, time course of cell numbers in culture was monitored at indicated time points and assessed by capillary-based CASY ${ }^{\circledR}$ cell counting. Data are presented as mean \pm SD ( $n=3$ in triplicates, nonlinear fit of exponential growth equation).

Abbreviations: mRNA, messenger RNA; NROB2, nuclear receptor subfamily 0 group B member 2; RT-PCR, real-time polymerase chain reaction; RCC-EW, renal cell carcinoma cell line; SD, standard deviation.

Monitoring the time course of cell numbers in the culture revealed that the doubling time of RCC-EW cells was markedly shorter than that of Caki-1 and Caki-2 cells: 29.9 hours (confidence interval [CI] 22.1 \pm 46.3 hours), 82.1 hours (CI 46.6 \pm 344.8 hours), and 45.9 hours (CI $29.3 \pm 105.5$ hours) (doubling time assessed by nonlinear fit of exponential growth equation, Figure 2B). Due to their enhanced proliferative activity, the RCC-EW cells were selected for further experiments.

\section{Impact of heterologous NROB2 on cell viability, proliferation, and cell cycle progression of RCC-EW cells}

Previous findings by Zhang et al suggest an impact of the NR on cell proliferation. ${ }^{13}$ To test whether NR0B2 exerts a similar influence on carcinoma cells derived from human kidney, the impact of adenoviral transfer of NR0B2 on RCC-EW cells was tested.

The heterologous transfer of NR0B2 was validated by Western blot analysis using different amounts of the NR0B2 adenovirus. The used adenoviral load was directly associated with the expression levels of NR0B2 protein (Figure 3A). Additionally, immunofluorescent staining of NR0B2 in RCC-EW cells was significantly enhanced after infection with Ad-NR0B2, but not after exposure to LacZ control (Ad-LacZ, Figure 3B). Importantly, H\&E staining revealed no significant change of cellular morphology after adenoviral infection with either Ad-NR0B2 or Ad-LacZ, although some cells transduced with NR0B2 appeared bigger and were swollen (Figure 3C).
Cell viability was monitored by quantification of resazurin turnover. As shown in Figure 4A, no significant difference was observed in metabolic activity after viral transfer of NR0B2 or LacZ. However, the effect of heterologous NR0B2 on proliferative activity determined by capillarybased cell counting over a period of 96 hours, revealed a significantly decelerated proliferation rate of RCC-EW cells after infection with the adenovirus encoding for the NR (total cell number 72 hours after infection with Ad-LacZ vs Ad-NR0B2 $9.2 \pm 4.6 \times 10^{5}$ vs $6.3 \pm 3.8 \times 10^{5}$; and 96 hours after infection with Ad-LacZ vs Ad-NR0B2 $11.5 \pm 8.7 \times 10^{5}$ vs $7.6 \pm 6.6 \times 10^{5}$; Figure 4B). Our results support the notion that NR0B2 overexpression also has an antiproliferative effect on renal cell carcinoma cells.

To qualify the impact of NR0B2 on cell cycle progression, flow cytometric analysis after propidium iodide staining of infected cells was performed. RCC-EW cells showed a pronounced shift of cell cycle phases 96 hours after adenoviral transfer of NR0B2 (Figure 4C). In detail, adenoviral overexpression of this NR significantly reduced the amount of cells passing the G1 phase (Ad-NR0B2 vs Ad-LacZ $77.8 \% \pm 0.1 \%$ vs $67.4 \% \pm 0.2 \%$ referring to total cell number, two-way analysis of variance, Dunnett's test post hoc, $P<0.0005$ ), while more cells were detected in the $\mathrm{S} / \mathrm{G} 2$ phase (Ad-NR0B2 vs Ad-LacZ $17.1 \% \pm 0.5 \%$ vs $24.7 \% \pm 0.4 \%$ referring to total cell number, two-way analysis of variance, Dunnett's test post hoc, $P<0.0005$ ).

It has previously been reported that the antiproliferative activity of NR0B2 in HCC is associated with a downregulation of the cell cycle regulator cyclin D1. ${ }^{17,18}$ To test whether 


\section{A}

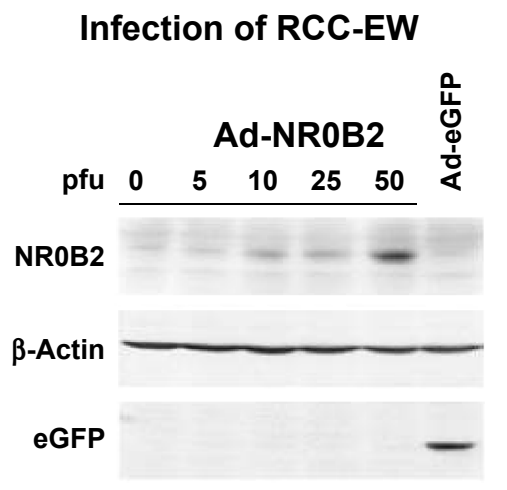

B

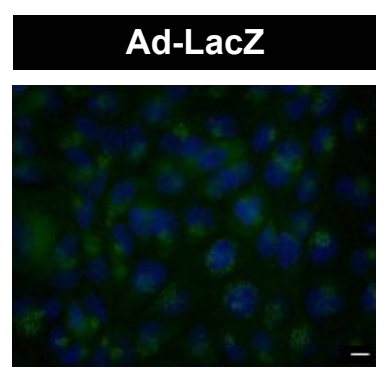

C

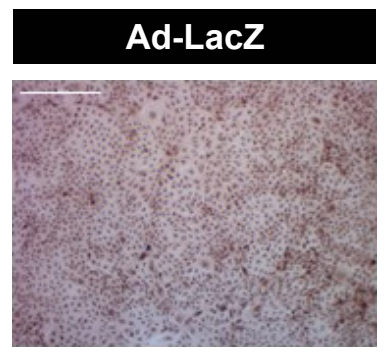

Ad-NR0B2

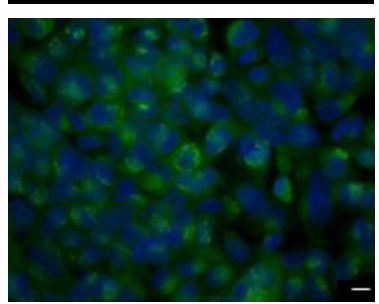

\section{Ad-NR0B2}

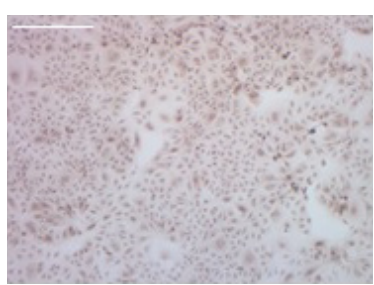

Figure 3 Validation of adenoviral transfer of NROB2 (Ad-NROB2) in RCC-EW cells.

Notes: (A) Western blot analysis of RCC-EW cell lysates 96 hours after infection with indicated amounts of pfu of Ad-NR0B2 or Ad-eGFP as control. (B) Immunofluorescent staining of RCC-EW cells with anti-NROB2 polyclonal primary antibody and Alexa Fluor ${ }^{\circledR} 488$-labeled secondary antibody (green) 12 hours after adenoviral transfer. Cell nuclei were counterstained with 4',6-diamidino-2-pheynylindole (blue). For infection of RCC-EW cells, 50 pfu per cell was used. Scale bar $=10 \mu \mathrm{m}$. (C) For assessment of cellular morphology, cells were fixed and stained with hematoxylin and eosin 96 hours after infection, Scale bar $=200 \mu \mathrm{m}$.

Abbreviations: pfu, plaque forming units; NROB2, nuclear receptor subfamily 0 group B member 2; RCC-EW, renal carcinoma cell line; eGFP, enhanced green fluorescent protein.

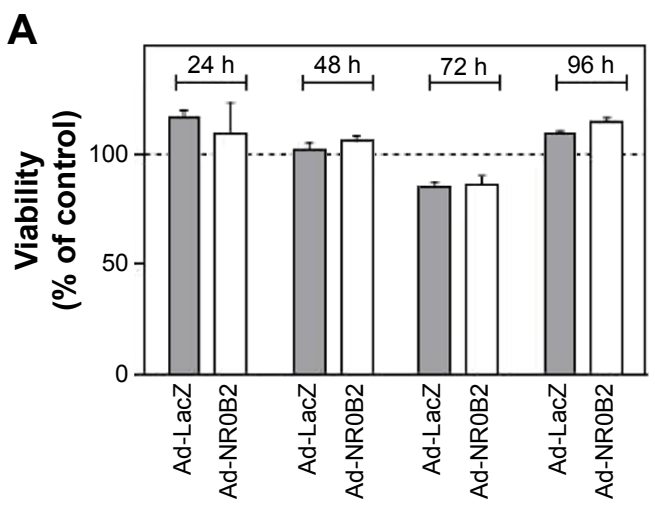

C

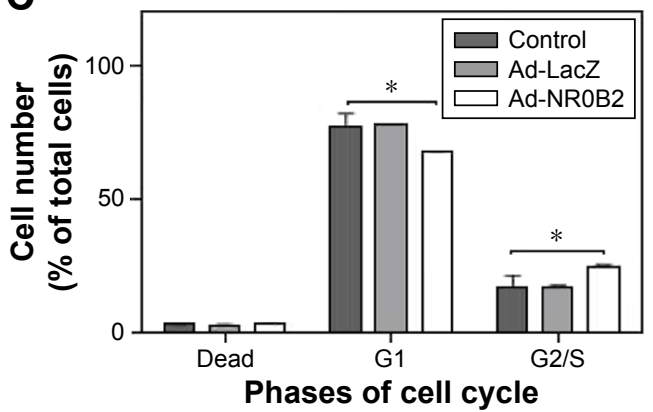

B

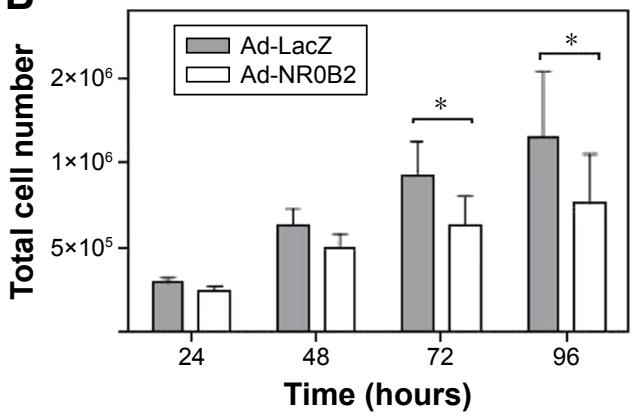

E

D

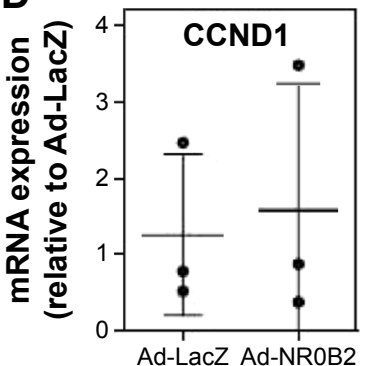

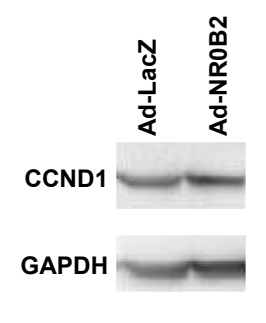

Figure 4 Heterologous NROB2 overexpression significantly decreased the proliferation of renal cell carcinoma cell line RCC-EW.

Notes: The impact of exogenous NROB2 overexpression on cell viability, proliferative activity, and cell cycle progression was analyzed. Fifty pfu per cell were used for infection with NROB2-adenovirus (Ad-NROB2) or LacZ-adenovirus as control (Ad-LacZ). Data from $\mathbf{A}$ to $\mathbf{D}$ are mean \pm SD. (A) Monitoring of fluorescence intensity of resazurin turnover ( $n=5, P>0.05$ by $t$-test). (B) Monitoring of the time course of cell count in culture at indicated time points assessed by capillary based $C A S Y{ }^{\circledR}$ cell counting $(n=5, * p<0.05$ by ratio paired $t$-test). (C) Cell cycle analysis by fluorescence-activated cell sorting ( $n=3, * P<0.05$ by two-way analysis of variance, Dunnett's test post hoc). (D) Impact of NROB2 on mRNA expression of cyclin DI (CCNDI) was assessed by TaqMan ${ }^{\circledR}$ quantitative RT-PCR. Data were obtained according to the $2^{-\triangle \Delta C t}$-method referring expression to that observed in cells infected with Ad-LacZ control ( $n=3$, not significant $P>0.05$ by Mann-Whitney test). (E) Cyclin DI protein expression assessed by Western blot analysis comparing RCC-EW cells in presence and absence of NROB2 adenovirus. GAPDH served as loading control.

Abbreviations: GAPDH, glyceraldehyde 3-phosphate dehydrogenase; NROB2, nuclear receptor subfamily 0 group B member 2; pfu, plaque forming units; RCC-EW, renal carcinoma cell line; RT-PCR, real-time polymerase chain reaction; SD, standard deviation; h, hours. 
cyclin D1 is also regulated by NR0B2 in renal carcinoma, the expression of cyclin D1 was assessed in RCC-EW cells in the presence and absence of NR0B2. However, neither mRNA (Figure 4D: $1.3 \pm 1.1$ vs $1.6 \pm 1.7, P=0.90$ ) nor protein expression (Figure 4E as assessed by Western blot analysis) were significantly altered by NR0B2.
Moreover, immunofluorescent staining of MKI67 revealed no significant change in the mean expression of this proliferation marker in the presence or absence of NR0B2 (mean of expression \pm SD referring to control: Ad-LacZ vs NR0B2 $1.00 \pm 0.50$ vs $1.05 \pm 0.24$, $t$-test, $P=0.85$; Figure 5).
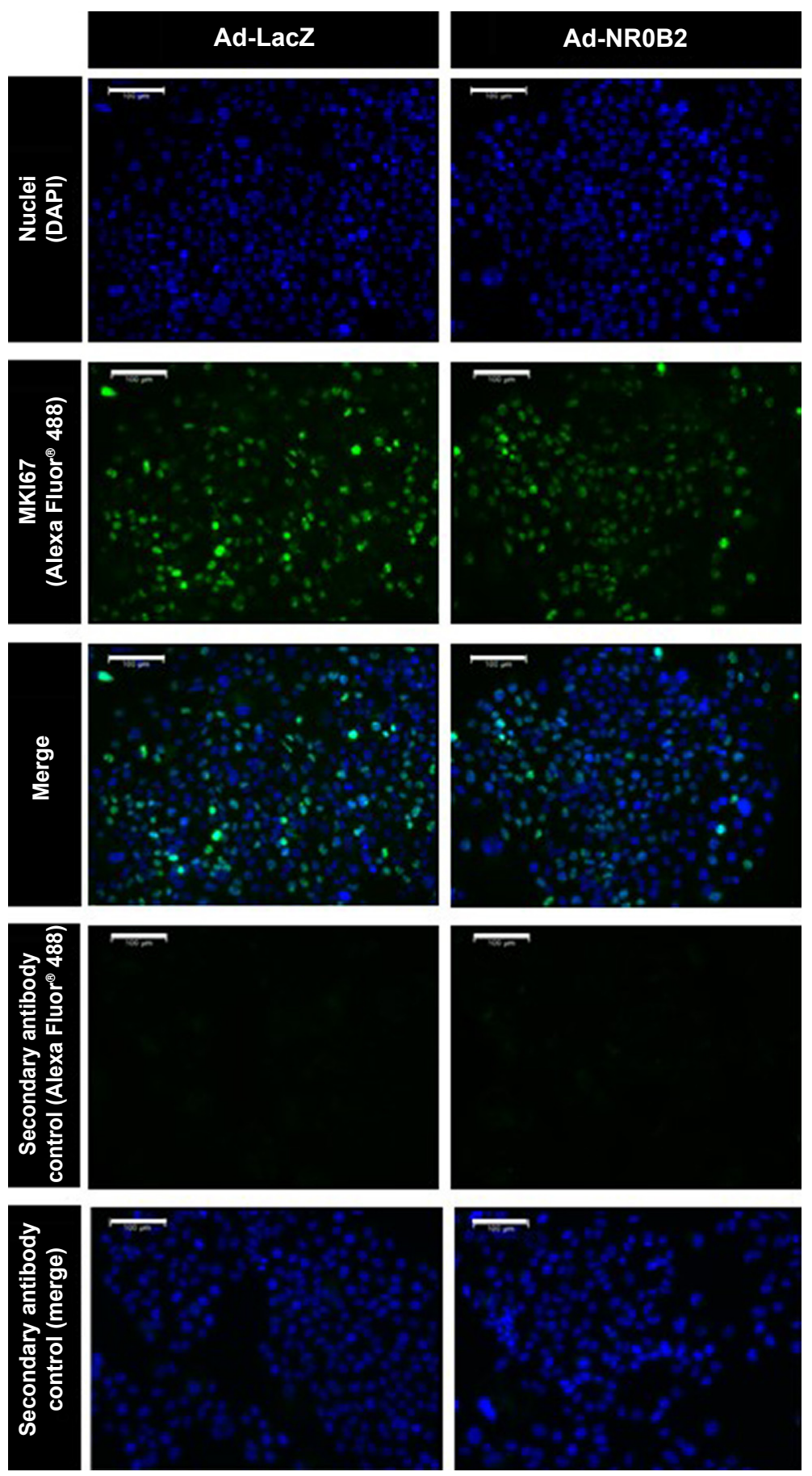

Figure 5 Immunofluorescent staining of MKI67 in RCC-EW cells.

Notes: Anti-MKI67 monoclonal primary antibody and Alexa Fluor ${ }^{\circledR} 488$-labeled secondary antibody (green) were used for staining of RCC-EW cells 96 hours after transduction with 50 plaque forming units of NROB2-adenovirus (Ad-NROB2) or LacZ-adenovirus (Ad-LacZ). Cell nuclei were counterstained with DAPI (blue). Scale bar $=100 \mu \mathrm{m}$. The micrograph is representative of three independent experiments.

Abbreviations: DAPI, 4',6-diamidino-2-pheynylindole; NROB2, nuclear receptor subfamily 0 group B member 2; RCC-EW, renal carcinoma cell line. 
A

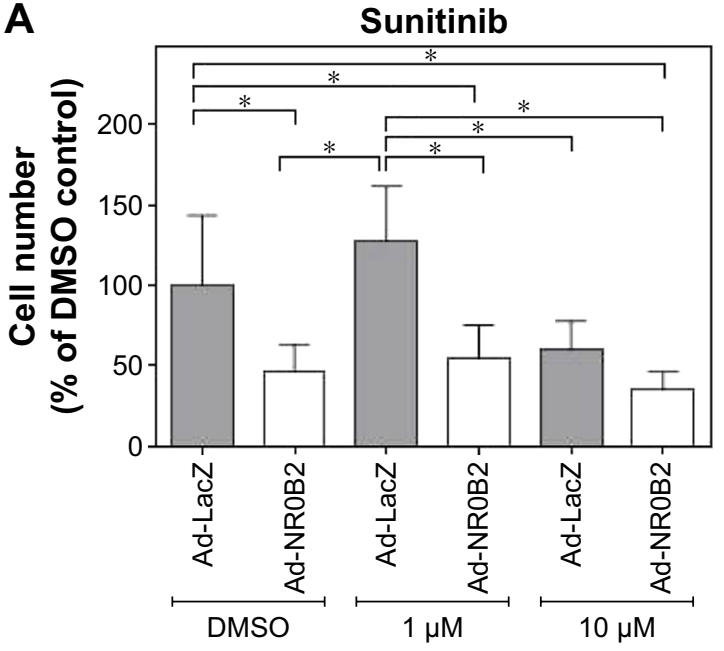

B

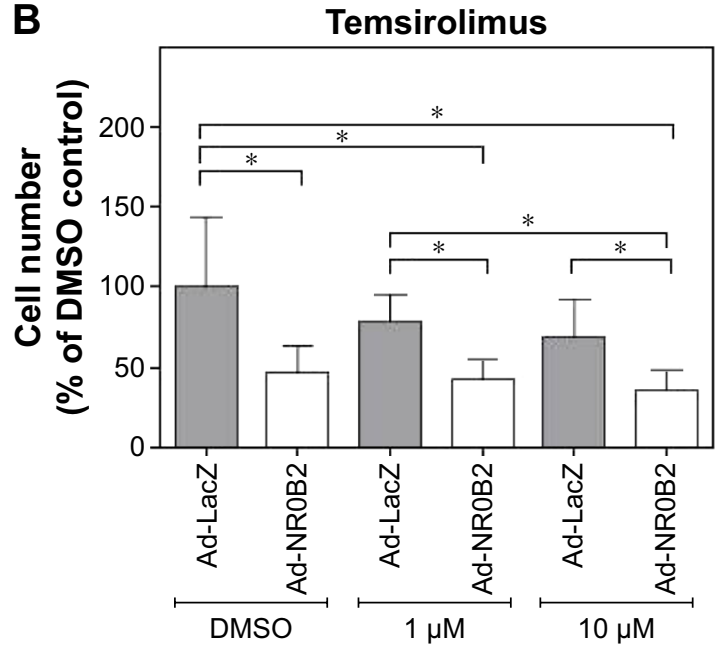

Figure 6 Impact of adenoviral transfer of NROB2 and antiproliferative compounds on RCC-EW cells.

Notes: The RCC-EW cells were infected with 50 plaque forming units of adenovirus containing LacZ or NROB2, respectively (Ad-LacZ, Ad-NR0B2), and were treated with different concentrations of $(\mathbf{A})$ sunitinib or $(\mathbf{B})$ temsirolimus. Cells were counted using capillary-based CASY ${ }^{\circledR}$ cell counter 96 hours after viral infection and 48 hours after application of compounds. Data are presented as mean $\pm S D\left(n=4,{ }^{*} P<0.05\right.$ by one-way analysis of variance, Tukey's posttest).

Abbreviations: DMSO, dimethyl sulfoxide; NROB2, nuclear receptor subfamily 0 group B member 2; SD, standard deviation; RCC-EW, renal carcinoma cell line.

\section{Impact of adenoviral expression of NROB2 on RCC-EW cells compared to chemotherapeutics}

Since NR0B2 significantly reduced cellular proliferation of renal carcinoma cells, we next compared the impact of heterologously overexpressed NR0B2 with the effectiveness of the tyrosine kinase inhibitor sunitinib or the mammalian target of rapamycin inhibitor temsirolimus, which are in clinical use for the treatment of renal cell carcinoma. ${ }^{19-21}$

When renal carcinoma cells RCC-EW not overexpressing NR0B2 were treated with sunitinib (Ad-LacZ/dimethyl sulfoxide [DMSO] vs Ad-LacZ/10 $\mu \mathrm{M}: 100 \% \pm 43.6 \%$ vs $60.0 \% \pm 17.8 \%$ ) or temsirolimus (Ad-LacZ/DMSO vs Ad-LacZ/10 $\mu \mathrm{M}: 100 \% \pm 43.6 \%$ vs $68.8 \% \pm 23.2 \%$ ), the impact of these compounds was limited to a slight but not statistically significant reduction in cell numbers compared to control cells treated with solvent control (Figure 6A and B). In contrast, a significant reduction in cell numbers was observed when cells were infected with NR0B2-adenovirus and simultaneously treated with DMSO $(47.0 \% \pm 16.4 \%)$, sunitinib (10 $\mu \mathrm{M}: 35.0 \% \pm 11.0 \%)$, or temsirolimus $(10 \mu \mathrm{M}$ : $36.3 \% \pm 12.6 \%$ ), indicating that infection with Ad-NR0B2 was more efficient than treatment with chemotherapeutics alone. However, inhibition of proliferation by a combination of adenoviral transfer and chemotherapeutic treatment was not stronger than usage of the adenovirus alone.

\section{Discussion}

Members of the transcription factor family of NRs have been reported to regulate the expression of target genes involved in cellular signaling, drug metabolism, drug transport, and/or tumor progression. Especially, NR0B2 has been addressed in terms of carcinogenesis. Studies using NR0B2 knockout mice demonstrate that the loss of this particular orphan NR results in enhanced malignant transformation and increased proliferation of hepatocytes, finally triggering the spontaneous development of HCC. ${ }^{10-13}$ Additionally, beneficial effects of farnesoid X receptor-induced NR0B2 expression on cell proliferation and tumor growth of liver cancer in nude mice have been observed. ${ }^{14}$ Notably, our results confirmed the previous findings by others and showed a significant reduction of NR0B2 expression when comparing the specimen of $\mathrm{HCC}$ and nonmalignant transformed tissue, which suggested that NR0B2 functions as tumor suppressor in this tissue..$^{12,13-16}$

The focus of our study was to show that downregulation or even loss of human NR0B2 is also characteristic for extrahepatic tumor entities. Yet, in our study, when using the commercially available cDNA panel, we observed difference in NR0B2 expression when comparing tumor and healthy control tissue samples of human non-small cell carcinoma of the lung. However, the results did not reach statistical significance, which may in part be explained by the small number of samples in the cDNA tumor panel used for screening. Nonetheless, Jeong et al previously identified mRNA expression of NR0B2 as a prognostic marker for the outcome of non-small cell lung cancer patients, thereby providing evidence for NR0B2 playing a role in tumor progression. ${ }^{22}$ Our results also indicated that NR0B2 expression is lower in samples of gastric adenocarcinoma, which might be in contrast to findings of Park et al, showing high 
NR0B2 expression in precancerous lesions of human gastric cancer. ${ }^{23}$ In our expression analysis using the commercially obtained cDNA panel, specimen of GIST showed even more pronounced downregulation of NR0B2 compared to healthy control samples. At this point, one might speculate that even though NR0B2 is highly expressed in intestinal metaplasia lesions of the stomach, its role in tumor progression in adenocarcinoma and GIST might be different.

Importantly, the findings of our expression studies screening the samples of the cDNA panel also revealed that NR0B2 is downregulated in human renal cell carcinoma compared to healthy tissue samples, suggesting that mechanisms of reduced NR0B2 expression may also play a role in this tumor entity. Our observation was further validated in a collection of renal carcinoma samples comprising a higher number of specimens from tumor and nonmalignant transformed tissue where each sample pair was derived from one individual. Since little is known about the role of NR0B2 in renal carcinogenesis, we further focused on NR0B2 in this tumor entity.

To assess the impact of NR0B2 on renal carcinoma cell proliferation, we performed an adenoviral transfer of NR0B2 in tumor kidney cells, thereby modulating the expression of this NR. The overexpression of NR0B2 in a human renal carcinoma cell line (RCC-EW) resulted in a significantly diminished proliferative activity in vitro.

Furthermore, some cells transduced with NR0B2 displayed cytoplasmic swelling, which is a characteristic of oncotic, nonapoptotic cell death due to disturbed intracellular ion homeostasis, and membrane integrity. ${ }^{24}$ Based on the fact that NR0B2 is also a transcriptional regulator of membrane transporters, ${ }^{8}$ it may be speculated that this NR is involved in the regulation of ion channels, thereby stabilizing the osmotic nature of the cell. Nonetheless, the growth-inhibiting effect of NR0B2 may also include other targets, which are involved in apoptosis and cell cycle progression. As reviewed by Zhang and Wang, these targets include NF- $\kappa$ B, P53, C-JUN, HDAC6, NUR77, and BCL2. ${ }^{12}$ Focusing on the latter, it is assumed that the growth inhibiting capacity of NR0B2 is based on the inhibitory interaction of this NR with BCL2, which is known to function as an antiapoptotic protein. The inhibition of BCL2 by NR0B2 promotes mitochondrial cytochrome c release and therefore apoptosis. ${ }^{12}$

Moreover, previous studies analyzing the role of NR0B2 in hepatocarcinogenesis show that the tumor-suppressing function of NR0B2 in HCC is based on the repression of cyclin D1 expression. In this regard, it is noteworthy that cyclin D1 is a positive regulator of cell cycle progression, ${ }^{25}$ and that cyclin D1 expression is assumed to be under the control of NR liver receptor homolog-1 (NR1H2). ${ }^{8}$ Thus the impact of NR0B2 in hepatocytes is assumed to be indirect, by negative modulation of nuclear receptor LHR-1. ${ }^{13,26,27}$ In accordance with this, the loss of NR0B2 in HCC is associated with increased cyclin D1 expression, which then promotes hepatocyte proliferation. ${ }^{13}$

Even though the NR0B2-dependent downregulation of cyclin D1 has been reported, the NR0B2-dependent impact on cyclin D1 expression, as previously observed in liver, was not found in RCC-EW cells, suggesting that additional regulators are involved, and that NR0B2-mediated cyclin D1 repression may be cell-specific. Although we did not observe a significant change in the expression of cyclin D1, there was a shift in cell cycle progression from G1 phase to S/G2 phase. In addition, our studies revealed no significant change in the expression of MKI67, which is a protein present during cell cycle progression, and which is absent in cells arrested in the G0 phase. ${ }^{28}$ These findings suggest that NR0B2 may rather trigger $\mathrm{G} 2$ arrest in renal carcinoma cells, preventing the cells from entering mitosis. Thus it needs to be clarified whether NR0B2 affects the expression or activity of proteins regulating $\mathrm{G} 2 / \mathrm{M}$ transition. ${ }^{29}$

Nevertheless, even if the underlying mechanism for the antiproliferative effect in renal carcinoma cells has not been clarified, our findings in human kidney cells, and the results of others showing the tumor growth inhibiting effect of NR0B2 in HCC, direct the attention to this NR as a target in cancer treatment. Focusing on this option, expression of NR0B2 may be influenced directly by heterologous overexpression of NR0B2, as realized by adenoviral transfer in our study. Importantly, in our in vitro studies, the direct impact of NR0B2 was more pronounced than that observed for chemotherapeutic treatment using tyrosine kinase inhibitor sunitinib or the mammalian target of rapamycin inhibitor temsirolimus, which are approved for the treatment of renal cell carcinoma. ${ }^{19}$ In this context, it is important that renal cell carcinoma exhibit pronounced chemoresistance, resulting in poor responsiveness to conventional chemotherapy. ${ }^{20,21}$

In this regard, it will be the focus of future studies to test potential therapeutic approaches, which could be followed to enhance NR0B2 activity. The first strategy could be ligand-mediated activation. However, this may be challenging, as NR0B2 is classified as an orphan receptor, since the ligands modulating its activity have not been identified so far, although some atypical retinoids are discussed as activating ligands. ${ }^{30}$ Another option to influence NR0B2 activity is the transcriptional modulation of $N R O B 2$ gene 
expression. It has been reported that NR0B2 is the target of ligand-activated NR, which bind to the highly conserved 5 -flanking promoter region of NR0B2 and induce the expression of this NR. Hence, affecting the activity of transcription factors, such as NR farnesoid X receptor or liver $\mathrm{X}$ receptor alpha, which are known regulators of NR0B2, may be another strategy to modulate NR0B2 expression. ${ }^{8,27,31}$ However, one has to consider that activation of NR may result in a high potential of adverse effects due to the fundamental role of NR in metabolic pathways and drug response, as we have learned from the lessons of pregnane $\mathrm{X}$ receptor-induced drug-drug interactions. ${ }^{8,9}$ A third approach to enhance the level of NR0B2 in tumors, and to compensate for an insufficient expression of this gene would be the use of gene therapy. Interestingly, the feasibility of viral gene transfer as therapeutic intervention has been proven in in vivo studies on heart failure, showing that adenoviral-transfer of sarcoendoplasmic reticulum calcium transport ATPase (SERCA2a) results in beneficial effects on cardiac myocyte contractility and calcium transport, and thereby on systolic and diastolic dysfunction. ${ }^{32}$

Taken together, our findings reveal that NR0B2 is downregulated in human renal cell carcinoma compared to healthy tissue, suggesting that mechanisms of reduced NR0B2 expression may play a role in the development and progression of this tumor entity. Our data also show that reconstitution of NR0B2 by adenoviral transfer diminishes cellular proliferation of human kidney tumor cells in vitro, supporting its regulatory function in renal cell cancer progression and its potential role in treatment of renal cell carcinoma.

\section{Disclosure}

The authors report no conflicts of interest in this work.

\section{References}

1. Burris TP, Busby SA, Griffin PR. Targeting orphan nuclear receptors for treatment of metabolic diseases and autoimmunity. Chem Biol. 2012;19(1):51-59.

2. Sherman MH, Downes M, Evans RM. Nuclear receptors as modulators of the tumor microenvironment. Cancer Prev Res. 2012;5(1):3-10.

3. Urquhart BL, Tirona RG, Kim RB. Nuclear receptors and the regulation of drug-metabolizing enzymes and drug transporters: implications for interindividual variability in response to drugs. J Clin Pharmacol. 2007; 47(5):566-578.

4. Seol W, Choi HS, Moore DD. An orphan nuclear hormone receptor that lacks a DNA binding domain and heterodimerizes with other receptors. Science. 1996;272(5266):1336-1339.

5. Kim MK, Chanda D, Lee IK, Choi HS, Park KG. Targeting orphan nuclear receptor SHP in the treatment of metabolic diseases. Expert Opin Ther Targets. 2010;14(4):453-466.

6. Bavner A, Sanyal S, Gustafsson JA, Treuter E. Transcriptional corepression by SHP: molecular mechanisms and physiological consequences. Trends Endocrinol Metab. 2005;16(10):478-488.
7. Johansson L, Bavner A, Thomsen JS, Farnegardh M, Gustafsson JA, Treuter E. The orphan nuclear receptor SHP utilizes conserved LXXLLrelated motifs for interactions with ligand-activated estrogen receptors. Mol Cell Biol. 2000;20(4):1124-1133.

8. Tirona RG, Kim RB. Nuclear receptors and drug disposition gene regulation. J Pharm Sci. 2005;94(6):1169-1186.

9. Meyer zu Schwabedissen HE, Kim RB. Hepatic OATP1B transporters and nuclear receptors PXR and CAR: interplay, regulation of drug disposition genes, and single nucleotide polymorphisms. Mol Pharm. 2009;6(6):1644-1661.

10. Baek SH, Kim KI. Emerging roles of orphan nuclear receptors in cancer. Annu Rev Physiol. 2014;76:177-195.

11. Zhang Y, Hagedorn CH, Wang L. Role of nuclear receptor SHP in metabolism and cancer. Biochim Biophys Acta. 2011;8:893-908.

12. Zhang Y, Wang L. Nuclear receptor small heterodimer partner in apoptosis signaling and liver cancer. Cancers. 2011;3(1):198-212.

13. Zhang Y, Xu P, Park K, Choi Y, Moore DD, Wang L. Orphan receptor small heterodimer partner suppresses tumorigenesis by modulating cyclin D1 expression and cellular proliferation. Hepatology. 2008;48(1):289-298.

14. Su H, Ma C, Liu J, et al. Downregulation of nuclear receptor FXR is associated with multiple malignant clinicopathological characteristics in human hepatocellular carcinoma. Am J Physiol Gastrointest Liver Physiol. 2012;303(11):G1245-G1253.

15. Park YY, Choi HS, Lee JS. Systems-level analysis of gene expression data revealed NR0B2/SHP as potential tumor suppressor in human liver cancer. Mol Cells. 2010;30(5):485-491.

16. He N, Park K, Zhang Y, Huang J, Lu S, Wang L. Epigenetic inhibition of nuclear receptor small heterodimer partner is associated with and regulates hepatocellular carcinoma growth. Gastroenterology. 2008; 134(3):793-802.

17. Botrugno OA, Fayard E, Annicotte JS, et al. Synergy between LRH-1 and beta-catenin induces $\mathrm{G} 1$ cyclin-mediated cell proliferation. Mol Cell. 2004;15(4):499-509.

18. Sato Y, Itoh F, Hareyama M, et al. Association of cyclin D1 expression with factors correlated with tumor progression in human hepatocellular carcinoma. J Gastroenterol. 1999;34(4):486-493.

19. Ljungberg B, Bensalah K, Canfield S, et al. EAU guidelines on renal cell carcinoma: 2014 update. Eur Urol. 2015;67(5):913-924.

20. Buczek M, Escudier B, Bartnik E, Szczylik C, Czarnecka A. Resistance to tyrosine kinase inhibitors in clear cell renal cell carcinoma: from the patient's bed to molecular mechanisms. Biochim Biophys Acta. 2014; 1845(1):31-41.

21. Santoni M, Pantano F, Amantini C, et al. Emerging strategies to overcome the resistance to current mTOR inhibitors in renal cell carcinoma. Biochim Biophys Acta. 2014;1845(2):221-231.

22. Jeong Y, Xie Y, Xiao G, et al. Nuclear receptor expression defines a set of prognostic biomarkers for lung cancer. PLoS Med. 2010;7(12): e1000378.

23. Park MJ, Kim KH, Kim HY, Kim K, Cheong J. Bile acid induces expression of COX-2 through the homeodomain transcription factor CDX1 and orphan nuclear receptor SHP in human gastric cancer cells. Carcinogenesis. 2008;29(12):2385-2393.

24. Kunzelmann K. Ion channels in regulated cell death. Cell Mol Life Sci. 2016;73(11-12):2387-2403.

25. Thomasova D, Anders HJ. Cell cycle control in the kidney. Nephrol Dial Transplant. 2015;30(10):1622-1630.

26. Oiwa A, Kakizawa T, Miyamoto T, et al. Synergistic regulation of the mouse orphan nuclear receptor SHP gene promoter by CLOCK-BMAL1 and LRH-1. Biochem Biophys Res Commun. 2007;353(4):895-901.

27. Goodwin B, Jones SA, Price RR, et al. A regulatory cascade of the nuclear receptors FXR, SHP-1, and LRH-1 represses bile acid biosynthesis. Mol Cell. 2000;6(3):517-526.

28. du Manoir S, Guillaud P, Camus E, Seigneurin D, Brugal G. Ki-67 labeling in postmitotic cells defines different Ki-67 pathways within the 2c compartment. Cytometry. 1991;12(5):455-463.

29. Stark GR, Taylor WR. Control of the G2/M transition. Mol Biotechnol. 2006;32(3):227-248. 
30. Chanda D, Park JH, Choi HS. Molecular basis of endocrine regulation by orphan nuclear receptor small heterodimer partner. Endocr J. 2008;55(2):253-268.

31. Goodwin B, Watson MA, Kim H, Miao J, Kemper JK, Kliewer SA. Differential regulation of rat and human CYP7A1 by the nuclear oxysterol receptor liver X receptor-alpha. Mol Endocrinol. 2003;17(3): 386-394.
32. Periasamy M. Adenoviral-mediated serca gene transfer into cardiac myocytes: how much is too much? Circ Res. 2001;88(4):373-375.

\section{Publish your work in this journal}

OncoTargets and Therapy is an international, peer-reviewed, open access journal focusing on the pathological basis of all cancers, potential targets for therapy and treatment protocols employed to improve the management of cancer patients. The journal also focuses on the impact of management programs and new therapeutic agents and protocols on

patient perspectives such as quality of life, adherence and satisfaction. The manuscript management system is completely online and includes a very quick and fair peer-review system, which is all easy to use. Visit http://www.dovepress.com/testimonials.php to read real quotes from published authors.

\footnotetext{
Submit your manuscript here: http://www.dovepress.com/oncotargets-and-therapy-journal
} 